\title{
The Teodorescu operator in Clifford analysis
}

\author{
F. Brackx* H. De Schepper*, M.E. Luna-Elizarrarás ${ }^{\ddagger}$ and M. Shapiro ${ }^{\ddagger}$ \\ * Clifford Research Group, Faculty of Engineering, Ghent University, Belgium \\ * Instituto Politécnico Nacional, Mexico City, Mexico
}

\begin{abstract}
Euclidean Clifford analysis is a higher dimensional function theory centred around monogenic functions, i.e. null solutions of a first order vector valued rotation invariant differential operator $\underline{\partial}$ called the Dirac operator. More recently, Hermitean Clifford analysis has emerged as a new branch, offering yet a refinement of the Euclidean case; it focusses on the simultaneous null solutions, called Hermitean monogenic functions, of two Hermitean Dirac operators $\partial_{\underline{z}}$ and $\partial_{z} \dagger$ which are invariant under the action of the unitary group. In Euclidean Clifford analysis the Teodorescu operator is the right inverse of the Dirac operator $\underline{\partial}$. In this paper Teodorescu operators for the Hermitean Dirac operators $\partial_{\underline{z}}$ and $\partial_{\underline{z}^{\dagger}}$ are constructed. Moreover the structure of the Euclidean and Hermitean Teodorescu operators is revealed by analyzing the more subtle behaviour of their components. Finally the obtained inversion relations are still refined for the differential operators issuing from the Euclidean and Hermitean Dirac operators by splitting the Clifford algebra product into its dot and wedge parts. Their relationship with several complex variables theory is discussed.
\end{abstract}

\section{Introduction}

In the complex plane the Teodorescu integral operator $T$ is well known. In a bounded domain $\Omega$ with smooth boundary $\partial \Omega$, its action on a function $u: \mathbb{R}^{2} \rightarrow \mathbb{C}$ is given by

$$
T[u](x, y)=\frac{1}{2 \pi i} \int_{\Omega} \frac{1}{\zeta-z} u(\zeta) d V(\xi, \eta), \quad \zeta=\xi+i \eta, z=x+i y
$$

It is the right inverse in $\Omega$ to the Cauchy-Riemann operator $\bar{\partial}$, i.e.: $\bar{\partial} T[u]=u$. The Teodorescu integral kernel thus coincides with the Cauchy kernel, which itself is a convolution kernel obtained by translating the fundamental solution $\frac{1}{2 \pi i} \frac{1}{z}$ of the Cauchy-Riemann operator $\bar{\partial}$.

In order to obtain an analogue of Teodorescu inversion in higher dimension, one first looks for a generalization of the Cauchy kernel. Now the Cauchy integral formula for holomorphic functions in the complex plane may be generalized to the case of several complex variables in two ways: either one takes a holomorphic kernel and an integral over the distinguished boundary $\partial_{0} \widetilde{D}=\prod_{j=1}^{n} \partial \widetilde{D}_{j}$ of a polydisk $\widetilde{D}=\prod_{j=1}^{n} \widetilde{D}_{j}$ in $\mathbb{C}^{n}$, leading to

$$
f\left(z_{1}, \ldots, z_{n}\right)=\frac{1}{(2 \pi i)^{n}} \int_{\partial_{0} \widetilde{D}} \frac{f\left(\xi_{1}, \ldots, \xi_{n}\right)}{\left(\xi_{1}-z_{1}\right) \cdots\left(\xi_{n}-z_{n}\right)} d \xi_{1} \wedge \cdots \wedge d \xi_{n}, z_{j} \in \stackrel{\circ}{D}_{j}
$$

or one takes an integral over the (piecewise) smooth boundary $\partial D$ of a bounded domain $D$ in $\mathbb{C}^{n}$, yielding

$$
f(z)=\int_{\partial D} f(\xi) U(\xi, z), \quad z \in \stackrel{\circ}{D}
$$

where, with $\cdot^{c}$ denoting the complex conjugate, the kernel

$U(\xi, z)=\frac{(n-1) !}{(2 \pi i)^{n}} \sum_{j=1}^{n}(-1)^{j-1} \frac{\xi_{j}^{c}-z_{j}^{c}}{|\xi-z|^{2 n}} d \xi_{1}^{c} \wedge \cdots \wedge d \xi_{j-1}^{c} \wedge d \xi_{j+1}^{c} \wedge \cdots \wedge d \xi_{n}^{c} \wedge d \xi_{1} \wedge \cdots \wedge d \xi_{n}$ 
is the so-called Martinelli-Bochner kernel, see e.g. [25], which is not holomorphic anymore but still harmonic. The history of formula (2), obtained independently by Martinelli and Bochner, has been described in detail in [24]. So we may expect that Teodorescu inversion in several complex variables will involve a harmonic integral kernel which will formally mimic the Martinelli-Bochner kernel. That this indeed is the case is explained in Section 5.

There is however an alternative for generalizing the two-dimensional Cauchy integral formula, offered by Clifford analysis, where functions defined in Euclidean space $\mathbb{R}^{2 n} \cong \mathbb{C}^{n}$ and taking values in a Clifford algebra are studied. The theory focusses on so-called monogenic functions, i.e. null solutions of the elliptic Dirac operator $\partial_{\underline{X}}$ factorizing the Laplace operator: $\partial_{\underline{X}}^{2}=-\Delta_{2 n}$. The Dirac operator being rotation invariant, the name Euclidean Clifford analysis is used nowadays to refer to this setting, see e.g. [11, 20, 22, 21]. Here the kernel appearing in the Clifford-Cauchy formula is monogenic, up to a pointwise singularity, while the integral is taken over the complete boundary:

$$
f(\underline{X})=\int_{\partial D} E(\underline{\Xi}-\underline{X}) d \sigma_{\Xi} f(\underline{\Xi}), \quad \underline{X} \in \stackrel{\circ}{D}
$$

with

$$
E(\underline{\Xi}-\underline{X})=\frac{1}{a_{2 n}} \frac{\bar{\Xi}-\underline{X}}{|\underline{\Xi}-\underline{X}|^{2 n}}
$$

$a_{2 n}=\frac{2^{n} \pi}{(n-1) !}$ being the area of the unit sphere $S^{2 n-1} \subset \mathbb{R}^{2 n} \cong \mathbb{C}^{n}$, - denoting the main conjugation in the Clifford algebra and $d \sigma_{\Xi}$ being a Clifford algebra valued differential form of order $(2 n-1)$. This Clifford-Cauchy integral formula has been a corner stone in the development of the function theory. The function $E(\underline{X})=\frac{1}{a_{2 n}} \frac{\bar{X}}{|X|^{2 n}}$ is the fundamental solution of the Euclidean Dirac operator $\partial_{\underline{X}}$, i.e. it holds in distributional sense that $\partial_{\underline{X}} E(\underline{X})=\delta(\underline{X})$, where $\delta(\underline{X})$ is the Dirac distribution in $\mathbb{R}^{2 n}$. As can be expected the Teodorescu inversion in Clifford analysis indeed involves a convolution with the Clifford-Cauchy kernel. This is recalled in Section 3 where also the underlying mechanisms leading to this result are revealed.

In a series of recent papers, Hermitean Clifford analysis has emerged as a refinement of the Euclidean case; it focusses on the simultaneous null solutions of the complex Hermitean Dirac operators $\partial_{\underline{z}}$ and $\partial_{\underline{z}^{\dagger}}$ which decompose the Laplacian in the sense that $4\left(\partial_{\underline{z}}+\partial_{\underline{z}^{\dagger}}\right)^{2}=$ $4\left(\partial_{\underline{z}} \partial_{\underline{z}^{\dagger}}+\partial_{\underline{z}^{\dagger}} \partial_{\underline{z}}\right)=\Delta_{2 n}$ and which are invariant under the action of the special unitary group. The study of complex Dirac operators was initiated in [27, 26, 28]. A systematic development of the function theory in the Hermitean Clifford analysis context, including the invariance properties with respect to the underlying Lie groups and Lie algebras, is still in full progress, see e.g. $[18,15,3,4,1,2]$. In this framework a Cauchy integral formula for Hermitean monogenic functions taking values in the complex Clifford algebra $\mathbb{C}_{2 n}$ or in complex spinor space $\mathbb{S}$ has been established in [10], and further integral representation formulae were developed in [16]. However, from the start it was clear that the desired formula could not have the traditional form of (1) or (2). Indeed, it is known (see [4]) that in the special case where the functions considered take their values in a specific part of spinor space, Hermitean monogenicity is equivalent with holomorphy in the underlying complex variables. It turned out that a matrix approach was the key to obtain the desired result. Moreover and as could be expected, the obtained Hermitean Cauchy integral formula reduces to the traditional Martinelli-Bochner formula (2) in the particular case mentioned. This also means that the theory of Hermitean monogenic functions not only refines Euclidean Clifford analysis (and thus harmonic analysis as well), but also has strong connections with the theory of functions of several complex variables, in some sense even encompassing its results.

In Section 4 we construct Hermitean Teodorescu inversion formulae not only for the Hermitean Dirac operators $\partial_{\underline{z}}$ and $\partial_{z^{\dagger}}$, but also for the associated differential operators $\partial_{\underline{z}} \bullet, \partial_{\underline{z}} \wedge$, $\partial_{z^{\dagger}} \bullet, \partial_{z^{\dagger}} \wedge$, obtained by splitting the Clifford algebra or geometric product into its "dot" and "wedge" parts. These associated differential operators are the counterparts in the multivector language of Clifford analysis of well-known differential operators for real and complex differential forms in Euclidean space.

As was already announced above, the results of this section are then interpreted, in Section 5 , for scalar valued functions of several complex variables, leading to the expected connections with the Martinelli-Bochner approach. 


\section{Preliminaries of Clifford analysis}

The real Clifford algebra $\mathbb{R}_{0, m}$ is constructed over the vector space $\mathbb{R}^{0, m}$ endowed with a non-degenerate quadratic form of signature $(0, m)$ and generated by the orthonormal basis $\left(e_{1}, \ldots, e_{m}\right)$. The non-commutative Clifford or geometric multiplication in $\mathbb{R}_{0, m}$ is governed by the rules

$$
e_{\alpha} e_{\beta}+e_{\beta} e_{\alpha}=-2 \delta_{\alpha \beta} \quad, \quad \alpha, \beta=1, \ldots, m
$$

As a basis for $\mathbb{R}_{0, m}$ one takes for any set $A=\left\{j_{1}, \ldots, j_{h}\right\} \subset\{1, \ldots, m\}$ the element $e_{A}=$ $e_{j_{1}} \ldots e_{j_{h}}$, with $1 \leq j_{1}<j_{2}<\cdots<j_{h} \leq m$, together with $e_{\emptyset}=1$, the identity element. Any Clifford number $a$ in $\mathbb{R}_{0, m}$ may thus be written as $a=\sum_{A} e_{A} a_{A}, a_{A} \in \mathbb{R}$, or still as $a=$ $\sum_{k=0}^{m}[a]_{k}$, where $[a]_{k}=\sum_{|A|=k} e_{A} a_{A}$ is the so-called $k$-vector part of $a$. Euclidean space $\mathbb{R}^{0, m}$ is embedded in $\mathbb{R}_{0, m}$ by identifying $\left(X_{1}, \ldots, X_{m}\right)$ with the Clifford vector $\underline{X}=\sum_{\alpha=1}^{m} e_{\alpha} X_{\alpha}$, for which it holds that $\underline{X}^{2}=-\langle\underline{X}, \underline{X}\rangle=-|\underline{X}|^{2}$. The Clifford or geometric product of two vectors splits into the sum of a scalar part, their commuting "dot"-product, and a 2-vector, or bivector, part, their anti-commuting "wedge"-product: $\underline{X} \underline{Y}=\underline{X} \bullet \underline{Y}+\underline{X} \wedge \underline{Y}$, with

$$
\begin{aligned}
& \underline{X} \bullet \underline{Y}=\sum_{\alpha=1}^{m} X_{\alpha} Y_{\alpha}=\frac{1}{2}(\underline{X} \underline{Y}+\underline{Y} \underline{X}) \\
& \underline{X} \wedge \underline{Y}=\sum_{\alpha<\beta} e_{\alpha} e_{\beta}\left(X_{\alpha} Y_{\beta}-Y_{\alpha} X_{\beta}\right)=\frac{1}{2}(\underline{X} \underline{Y}-\underline{Y} \underline{X})
\end{aligned}
$$

The Fischer dual of $\underline{X}$ is the vector valued Dirac operator $\partial_{\underline{X}}=\sum_{\alpha=1}^{m} e_{\alpha} \partial_{X_{\alpha}}$, underlying the notion of monogenicity of a function, a notion which is the higher dimensional counterpart of holomorphy in the complex plane. More explicitly a function $f$ defined and continuously differentiable in an open region $\Omega$ of $\mathbb{R}^{m}$ and taking values in $\mathbb{R}_{0, m}$ is called (left) monogenic in $\Omega$ if $\partial_{\underline{X}}[f]=0$ in $\Omega$. As the Dirac operator factorizes the Laplacian: $\Delta_{m}=-\partial_{\underline{X}}^{2}$, monogenicity can be regarded as a refinement of harmonicity. We refer to this setting as the Euclidean case, since the fundamental group leaving the Dirac operator $\partial_{\underline{X}}$ invariant is the special orthogonal group $\mathrm{SO}(m ; \mathbb{R})$, which is doubly covered by the $\operatorname{Spin}(m)$ group of the Clifford algebra $\mathbb{R}_{0, m}$. For this reason, the Dirac operator $\partial_{\underline{X}}$ is also called rotation invariant.

When allowing for complex constants and taking the dimension to be even: $m=2 n$, the generators $\left(e_{1}, \ldots, e_{2 n}\right)$, still satisfying the multiplication rules $(3)$, produce the complex Clifford algebra $\mathbb{C}_{2 n}=\mathbb{R}_{0,2 n} \oplus i \mathbb{R}_{0,2 n}$. Any complex Clifford number $\lambda \in \mathbb{C}_{2 n}$ may thus be written as $\lambda=a+i b, a, b \in \mathbb{R}_{0,2 n}$, an observation leading to the definition of the Hermitean conjugation $\lambda^{\dagger}=(a+i b)^{\dagger}=\bar{a}-i \bar{b}$, where the bar notation stands for the usual Clifford conjugation in $\mathbb{R}_{0,2 n}$, i.e. the main anti-involution for which $\bar{e}_{\alpha}=-e_{\alpha}, \alpha=1, \ldots, 2 n$. This Hermitean conjugation also leads to a Hermitean inner product and its associated norm on $\mathbb{C}_{2 n}$ given by $(\lambda, \mu)=\left[\lambda^{\dagger} \mu\right]_{0}$ and $|\lambda|=\sqrt{\left[\lambda^{\dagger} \lambda\right]_{0}}=\left(\sum_{A}\left|\lambda_{A}\right|^{2}\right)^{1 / 2}$. This is the framework for so-called Hermitean Clifford analysis, a refinement of Euclidean Clifford analysis.

An elegant way of introducing it consists in considering a complex structure, i.e. a specific $\mathrm{SO}(2 n ; \mathbb{R})$-element $J$ for which $J^{2}=\mathbf{- 1}$ (see $\left.[3,4]\right)$. Here, $J$ is chosen to act upon the generators $e_{1}, \ldots, e_{2 n}$ of the Clifford algebra as $J\left[e_{j}\right]=-e_{n+j}$ and $J\left[e_{n+j}\right]=e_{j}, j=1, \ldots, n$. With $J$ one associates two projection operators $\frac{1}{2}(\mathbf{1} \pm i J)$ which produce the main objects of the Hermitean setting by acting upon the corresponding ones in the Euclidean framework. First, the so-called Witt basis elements $\left(\mathfrak{f}_{j}, \mathfrak{f}_{j}^{\dagger}\right)_{j=1}^{n}$ for $\mathbb{C}_{2 n}$ are obtained from the orthogonal basis elements $e_{\alpha}$ :

$$
\begin{aligned}
\mathfrak{f}_{j}=\frac{1}{2}(\mathbf{1}+i J)\left[e_{j}\right]=\frac{1}{2}\left(e_{j}-i e_{n+j}\right), \quad j=1, \ldots, n \\
\mathfrak{f}_{j}^{\dagger}=-\frac{1}{2}(\mathbf{1}-i J)\left[e_{j}\right]=-\frac{1}{2}\left(e_{j}+i e_{n+j}\right), \quad j=1, \ldots, n
\end{aligned}
$$

The Witt basis elements satisfy the respective Grassmann and duality identities

$$
\begin{aligned}
& \mathfrak{f}_{j} \mathfrak{f}_{k}+\mathfrak{f}_{k} \mathfrak{f}_{j}=\mathfrak{f}_{j}^{\dagger} \mathfrak{f}_{k}^{\dagger}+\mathfrak{f}_{k}^{\dagger} \mathfrak{f}_{j}^{\dagger}=0, \quad j, k=1, \ldots, n \\
& \mathfrak{f}_{j} \mathfrak{f}_{k}^{\dagger}+\mathfrak{f}_{k}^{\dagger} \mathfrak{f}_{j}=\delta_{j k}, \quad j, k=1, \ldots, n
\end{aligned}
$$


including their isotropy. A vector in $\mathbb{R}^{0,2 n}$ is now denoted by $\left(x_{1}, \ldots, x_{n}, y_{1}, \ldots, y_{n}\right)$ and identified with the Clifford vector

$$
\underline{X}=\sum_{j=1}^{n}\left(e_{j} x_{j}+e_{n+j} y_{j}\right)
$$

The action of the complex structure $J$ on $\underline{X}$ yields

$$
\underline{X} \mid=J[\underline{X}]=\sum_{j=1}^{n}\left(e_{j} y_{j}-e_{n+j} x_{j}\right)
$$

the so-called twisted vector. The Clifford vectors $\underline{X}$ and $\underline{X} \mid$ anti-commute, since they are orthogonal w.r.t. the standard Euclidean scalar product. More precisely, see also [17]:

Lemma 1 One has

$$
\begin{aligned}
& \text { (i) } \underline{X} \bullet \underline{X} \mid=0 \\
& \text { (ii) } \underline{X} \wedge \underline{X} \mid=\sum_{j \neq k} x_{j} y_{k}\left(e_{j} e_{k}-e_{n+k} e_{n+j}\right)-\sum_{j, k} e_{j} e_{n+k}\left(x_{j} x_{k}+y_{j} y_{k}\right) \\
& \text { (iii) } \underline{X} \mid \wedge \underline{X}=\sum_{j \neq k} x_{j} y_{k}\left(e_{k} e_{j}-e_{n+j} e_{n+k}\right)-\sum_{j, k} e_{n+k} e_{j}\left(x_{j} x_{k}+y_{j} y_{k}\right) \\
& \text { (iv) } \underline{X} \underline{X}|+\underline{X}| \underline{X}=\underline{X} \wedge \underline{X}|+\underline{X}| \wedge \underline{X}=0
\end{aligned}
$$

The actions of the projection operators on the Clifford vector $\underline{X}$ then produce the conjugate Hermitean Clifford variables $\underline{z}$ and $\underline{z}^{\dagger}$ :

$$
\begin{aligned}
& \underline{z}=\frac{1}{2}(\mathbf{1}+i J)[\underline{X}]=\frac{1}{2}(\underline{X}+i \underline{X} \mid)=\sum_{j=1}^{n} \mathfrak{f}_{j} z_{j} \\
& \underline{z}^{\dagger}=-\frac{1}{2}(\mathbf{1}-i J)[\underline{X}]=-\frac{1}{2}(\underline{X}-i \underline{X} \mid)=\sum_{j=1}^{n} \mathfrak{f}_{j}^{\dagger} z_{j}^{c}
\end{aligned}
$$

where $n$ complex variables $z_{j}=x_{j}+i y_{j}$ have been introduced, with complex conjugates $z_{j}^{c}=x_{j}-i y_{j}, j=1, \ldots, n$. Finally, the Hermitean Dirac operators $\partial_{\underline{z}}$ and $\partial_{\underline{z}^{\dagger}}$ are obtained from the Euclidean Dirac operator $\partial_{\underline{X}}$ :

$$
\begin{aligned}
& \partial_{\underline{z}^{\dagger}}=\frac{1}{4}(\mathbf{1}+i J)\left[\partial_{\underline{X}}\right]=\frac{1}{4}\left(\partial_{\underline{X}}+i \partial_{\underline{X} \mid}\right)=\sum_{j=1}^{n} f_{j}^{\dagger} \partial_{z_{j}} \\
& \partial_{\underline{z}}=-\frac{1}{4}(\mathbf{1}-i J)\left[\partial_{\underline{X}}\right]=-\frac{1}{4}\left(\partial_{\underline{X}}-i \partial_{\underline{X} \mid}\right)=\sum_{j=1}^{n} \mathfrak{f}_{j} \partial_{z_{j}^{c}}
\end{aligned}
$$

involving the classical Cauchy-Riemann operators $\partial_{z_{j}^{c}}=\frac{1}{2}\left(\partial_{x_{j}}+i \partial_{y_{j}}\right)$ and their complex conjugates $\partial_{z_{j}}=\frac{1}{2}\left(\partial_{x_{j}}-i \partial_{y_{j}}\right)$ in the complex $z_{j}$-planes, $j=1, \ldots, n$. Here also the so-called twisted Dirac operator

$$
\partial_{\underline{X} \mid}=J\left[\partial_{\underline{X}}\right]=\sum_{j=1}^{n}\left(e_{j} \partial_{y_{j}}-e_{n+j} \partial_{x_{j}}\right)
$$

arises. As is the case for $\partial_{\underline{X}}$, a notion of (twisted) monogenicity may be associated in a natural way to $\partial_{\underline{X}} \mid$ as well.

The Hermitean vector variables and Dirac operators are isotropic, i.e.

$$
(\underline{z})^{2}=\left(\underline{z}^{\dagger}\right)^{2}=0 \quad \text { and } \quad\left(\partial_{\underline{z}}\right)^{2}=\left(\partial_{\underline{z}^{\dagger}}\right)^{2}=0
$$

whence the Laplacian $\Delta_{2 n}=-\partial_{\underline{X}}^{2}=-\partial_{\underline{X}}^{2}$ allows for the decomposition and factorization

$$
\Delta_{2 n}=4\left(\partial_{\underline{z}} \partial_{\underline{z}^{\dagger}}+\partial_{\underline{z}^{\dagger}} \partial_{\underline{z}}\right)=4\left(\partial_{\underline{z}}+\partial_{\underline{z}^{\dagger}}\right)^{2}
$$

while also

$$
\left(\underline{z}+\underline{z}^{\dagger}\right)^{2}=\underline{z} \underline{z}^{\dagger}+\underline{z}^{\dagger} \underline{z}=|\underline{z}|^{2}=\left|\underline{z}^{\dagger}\right|^{2}=|\underline{X}|^{2}=|\underline{X}|^{2}
$$


A continuously differentiable function $g$ on an open region $\Omega$ of $\mathbb{R}^{2 n}$ with values in the complex Clifford algebra $\mathbb{C}_{2 n}$ is called (left) Hermitean monogenic (or $\mathrm{h}$-monogenic) in $\Omega$ if and only if it simultaneously is $\partial_{X}-$ and $\partial_{X}-$ monogenic in $\Omega$, i.e. it satisfies in $\Omega$ the system $\partial_{\underline{X}} g=0=\partial_{\underline{X}} g$ or the equivalent system $\partial_{\underline{z}} g=0=\partial_{\underline{z} \dagger} g$. It remains to recall the group invariance underlying this system. To that end we consider the group $\widetilde{\mathrm{U}}(n) \subset \operatorname{Spin}(2 n)$, given by

$$
\widetilde{\mathrm{U}}(n)=\{s \in \operatorname{Spin}(2 n) \mid \exists \theta \geq 0: \bar{s} I=\exp (-i \theta) I\}
$$

its definition involving the self-adjoint primitive idempotent $I=I_{1} \ldots I_{n}$ with $I_{j}=\mathfrak{f}_{j} \mathrm{f}_{j}^{\dagger}=$ $\frac{1}{2}\left(1-i e_{j} e_{n+j}\right), j=1, \ldots, n$. It has been proved, see [18], that this group constitutes a realisation in the Clifford algebra of the unitary group $\mathrm{U}(n)$, and moreover, that its action leaves the Hermitean Dirac operators invariant. Less precisely, one thus says that the operators as well as the notion of $\mathrm{h}-$ monogenicity are invariant under the action of the unitary group.

\section{The Euclidean Teodorescu operators}

Let $\Omega$ be a fixed open bounded domain in $\mathbb{R}^{2 n} \cong \mathbb{C}^{n}$ with smooth boundary $\partial \Omega$. It is wellknown that the right inverses of the Euclidean Dirac operators $\partial_{\underline{X}}$ and $\partial_{\underline{X}}$ are the Teodorescu operators $T$ and $T \mid: C_{1}(\Omega) \rightarrow C_{1}(\Omega)$, given by

$$
\begin{aligned}
T[u](\underline{X}) & =-\int_{\Omega} E(\underline{Y}-\underline{X}) u(\underline{Y}) d V(\underline{Y}) \\
T \mid[u](\underline{X}) & =-\int_{\Omega} E \mid(\underline{Y}-\underline{X}) u(\underline{Y}) d V(\underline{Y})
\end{aligned}
$$

where

$$
\begin{aligned}
E(\underline{X}) & =\frac{1}{a_{2 n}} \frac{\bar{X}}{|\underline{X}|^{2 n}}=-\frac{1}{a_{2 n}} \frac{\underline{X}}{r^{2 n}}=\sum_{\alpha=1}^{2 n}\left(-\frac{1}{a_{2 n}} \frac{X_{\alpha}}{r^{2 n}}\right) e_{\alpha}=\sum_{\alpha=1}^{2 n} E_{\alpha} e_{\alpha} \\
& =\sum_{j=1}^{n}\left(-\frac{1}{a_{2 n}} \frac{x_{j}}{r^{2 n}}\right) e_{j}+\left(-\frac{1}{a_{2 n}} \frac{y_{j}}{r^{2 n}}\right) e_{n+j}=\sum_{j=1}^{n} E_{j} e_{j}+E_{n+j} e_{n+j} \\
E \mid(\underline{X}) & =\frac{1}{a_{2 n}} \frac{\underline{X} \mid}{|\underline{X}|^{2 n}}=-\frac{1}{a_{2 n}} \frac{\underline{X} \mid}{r^{2 n}}=\sum_{\alpha=1}^{2 n}\left(-\frac{1}{a_{2 n}} \frac{\left.X\right|_{\alpha}}{r^{2 n}}\right) e_{\alpha}=\left.\sum_{\alpha=1}^{2 n} E\right|_{\alpha} e_{\alpha} \\
& =\sum_{j=1}^{n}\left(-\frac{1}{a_{2 n}} \frac{y_{j}}{r^{2 n}}\right) e_{j}-\left(-\frac{1}{a_{2 n}} \frac{x_{j}}{r^{2 n}}\right) e_{n+j}=\sum_{j=1}^{n} E_{n+j} e_{j}-E_{j} e_{n+j}
\end{aligned}
$$

are the fundamental solutions of the respective Euclidean Dirac operators considered, which means that in distributional sense $\partial_{\underline{X}} E(\underline{X})=\delta(\underline{X})$ and $\partial_{\underline{X} \mid} E \mid(\underline{X})=\delta(\underline{X} \mid)=\delta(\underline{X})$, where $\delta$ stands for the Dirac distribution in $\mathbb{R}^{2 n}$. It thus holds that for each function $u \in C_{1}(\Omega)$ and each $\underline{X} \in \Omega$

which also can be written as

$$
\partial_{\underline{X}} T[u](\underline{X})=u(\underline{X}), \quad \partial_{\underline{X}} T \mid[u](\underline{X})=u(\underline{X})
$$

$$
\partial_{\underline{X}} T=\mathbf{1}_{\Omega}, \quad \partial_{\underline{X}}|T|=\mathbf{1}_{\Omega}
$$

As a matter of fact this result is quite remarkable since the Euclidean Dirac operators and the corresponding Teodorescu operators are Clifford vector valued, implying that the actions of $\partial_{\underline{X}}$ on $T$ and of $\partial_{X}$ on $T \mid$ are expected to be para-bivector valued, i.e. resulting in a sum of a scalar or "dot" and a bivector or "wedge" part. As the function $u$ may be chosen in particular to be scalar valued, it follows that it must hold that

$$
\partial_{\underline{X}} \bullet T=\mathbf{1}_{\Omega}, \quad \partial_{\underline{X} \mid} \bullet T \mid=\mathbf{1}_{\Omega}
$$

while

$$
\partial_{\underline{X}} \wedge T=0, \quad \partial_{\underline{X}} \wedge T \mid=0
$$


In other words the Teodorescu operators $T$ and $T \mid$ are right inverses to the differential operators $\partial_{\underline{X}} \bullet$ and $\partial_{\underline{X}} \bullet$, which are the Clifford analysis counterparts to the well known operators of exterior co-derivative $d^{*}$ and its twisted counterpart $\left.d\right|^{*}$ for smooth differential forms in Euclidean space. The counterparts of the exterior derivative $d$ and its twisted version $d \mid$ are the respective operators $\partial_{\underline{X}} \wedge$ and $\partial_{\underline{X}} \mid \wedge$, which apparently are annihilating the respective Teodorescu operators. For an overview of the similarities between real and complex differential forms in open subsets of $\mathbb{R}^{m}$ and $\mathbb{C}^{n}$ on the one hand and multivector functions in Euclidean and Hermitean Clifford analysis on the other hand, we refer the reader to [14, 19].

For a better understanding of the meaning of (4)-(6) we will now consider the derivatives of the components of the Teodorescu operators. The expressions obtained involve the multidimensional Fp-distributions "finite part" which are defined using the Fp-distribution on the real line; for a thorough study of these and related families of distributions in Clifford analysis we refer the reader to $[12,13,5,6,7,8,9]$. Restricting our considerations to the operator $T$, we put $T=\sum_{\alpha=1}^{m} e_{\alpha} T_{\alpha}$, where

$$
T_{\alpha}[u](\underline{X})=-\int_{\partial \Omega} E_{\alpha}(\underline{Y}-\underline{X}) u(\underline{Y}) d V(\underline{Y}), \quad \alpha=1, \ldots, m
$$

As an auxiliary result we need the following formulae concerning the distributional derivatives of the components $E_{\alpha}(\alpha=1, \ldots, m)$ of the fundamental solution $E$ (see [17]). Note that in the present context we have $m=2 n$; however, when considering only the operator $T$, the results below remain valid for general dimension $m$.

Lemma 2 In distributional sense one has

(i) $\partial_{X_{\beta}} E_{\alpha}=\frac{m}{a_{m}} \mathrm{Fp} \frac{X_{\alpha} X_{\beta}}{r^{m+2}}$, for all $\alpha \neq \beta, \alpha, \beta=1, \ldots, m$;

(ii) $\partial_{X_{\alpha}} E_{\alpha}=\frac{m}{a_{m}} \mathrm{Fp} \frac{X_{\alpha}^{2}}{r^{m+2}}-\frac{1}{m} \mathrm{Fp} \frac{1}{r^{m}}-\frac{1}{m} \delta$, for all $\alpha=1, \ldots, m$.

Proposition 1 For $u \in C_{1}(\bar{\Omega})$ and $\underline{X} \in \Omega$ one has

$$
\begin{aligned}
& \partial_{X_{\alpha}} T_{\alpha}[u](\underline{X})=-\frac{1}{m} u(\underline{X})-\frac{1}{a_{m}} \operatorname{Fp} \int_{\Omega} \frac{u(\underline{Y})}{|\underline{X}-\underline{Y}|^{m}} d V(\underline{Y})+\frac{m}{a_{m}} \operatorname{Fp} \int_{\Omega} \frac{\left(X_{\alpha}-Y_{\alpha}\right)^{2}}{|\underline{X}-\underline{Y}|^{m+2}} u(\underline{Y}) d V(Y) \\
& \partial_{X_{\beta}} T_{\alpha}[u](\underline{X})=\frac{m}{a_{m}} \operatorname{Fp} \int_{\Omega} \frac{\left(X_{\alpha}-Y_{\alpha}\right)\left(X_{\beta}-Y_{\beta}\right)}{|\underline{X}-\underline{Y}|^{m+2}} u(\underline{Y}) d V(\underline{Y})
\end{aligned}
$$

Proof.

Let the function $u \in C_{1}(\bar{\Omega})$ be extended by zero to $\mathbb{R}^{m} \backslash \bar{\Omega}$, this extension $\widetilde{u}$ being interpreted as a compactly supported distribution. Then one has

$$
T_{\alpha}[\widetilde{u}](\underline{X})=\int_{\Omega} E_{\alpha}(\underline{X}-\underline{Y}) \widetilde{u}(\underline{Y}) d V(\underline{Y})=E_{\alpha} * \widetilde{u}(\underline{X})
$$

and hence, using the formulae of Lemma 2

$$
\partial_{X_{\alpha}} T_{\alpha}[\widetilde{u}]=\partial_{X_{\alpha}} E_{\alpha} * \widetilde{u}=\left(\frac{m}{a_{m}} \mathrm{Fp} \frac{X_{\alpha}^{2}}{r^{m+2}}-\frac{1}{m} \mathrm{Fp} \frac{1}{r^{m}}-\frac{1}{m} \delta\right) * \widetilde{u}
$$

Restricting to $\underline{X} \in \Omega$ formula (7) is readily obtained. One also has

$$
\partial_{X_{\beta}} T_{\alpha}[\widetilde{u}]=\partial_{X_{\beta}} E_{\alpha} * \widetilde{u}=\left(\frac{m}{a_{m}} \operatorname{Fp} \frac{X_{\alpha} X_{\beta}}{r^{m+2}}\right) * \widetilde{u}
$$

yielding formula (8).

Remark 1 Formulae (7) and (8) may also be written as

$$
\begin{aligned}
& \partial_{X_{\alpha}} \int_{\Omega} E_{\alpha}(\underline{Y}-\underline{X}) u(\underline{Y}) d V(\underline{Y})=\frac{1}{m} u(\underline{X})+\mathrm{pv} \int_{\Omega} \partial_{X_{\alpha}} E_{\alpha}(\underline{Y}-\underline{X}) u(\underline{Y}) d V(\underline{Y}) \\
& \partial_{X_{\beta}} \int_{\Omega} E_{\alpha}(\underline{Y}-\underline{X}) u(\underline{Y}) d V(\underline{Y})=\operatorname{pv} \int_{\Omega} \partial_{X_{\beta}} E_{\alpha}(\underline{Y}-\underline{X}) u(\underline{Y}) d V(\underline{Y})
\end{aligned}
$$

where pv stands for the "Cauchy principal value" of the considered integral. 
Remark 2 For the technique used in the above proof the condition $u \in C_{1}(\bar{\Omega})$ is not really necessary; it suffices to have e.g. $u \in C_{1}(\Omega) \cap L_{1}^{\text {loc }}(\Omega)$. In fact the results may be obtained for functions $u \in C_{1}(\Omega)$ but then an approach such as in e.g. [23] is needed.

Corollary 1 For $u \in C_{1}(\bar{\Omega})$ and $\underline{X} \in \Omega$ one has

$$
\partial_{X_{\beta}} T[u](\underline{X})=-\frac{1}{m} e_{\beta} u(\underline{X})-\operatorname{pv} \int_{\Omega} \partial_{X_{\beta}} E(\underline{Y}-\underline{X}) u(\underline{Y}) d V(\underline{Y})
$$

Proof.

Using the results of Proposition 1 one obtains

$$
\begin{aligned}
\partial_{X_{\beta}} T[u](\underline{X})= & \sum_{\alpha=1}^{m} e_{\alpha} \partial_{X_{\beta}} T_{\alpha}[u](\underline{X})=e_{\beta} \partial_{X_{\beta}} T_{\beta}[u](\underline{X})+\sum_{\alpha \neq \beta} e_{\alpha} \partial_{X_{\beta}} T_{\alpha}[u](\underline{X}) \\
= & -e_{\beta}\left(\frac{1}{m} u(\underline{X})+\mathrm{pv} \int_{\Omega} \partial_{X_{\beta}} E_{\beta}(\underline{Y}-\underline{X}) u(\underline{Y}) d V(\underline{Y})\right) \\
& -\sum_{\alpha \neq \beta} e_{\alpha}\left(\mathrm{pv} \int_{\Omega} \partial_{X_{\beta}} E_{\alpha}(\underline{Y}-\underline{X}) u(\underline{Y}) d V(\underline{Y})\right) \\
= & -\frac{1}{m} e_{\beta} u(\underline{X})-\operatorname{pv} \int_{\Omega} \partial_{X_{\beta}} \sum_{\alpha=1}^{m} e_{\alpha} E_{\alpha}(\underline{Y}-\underline{X}) u(\underline{Y}) d V(\underline{Y})
\end{aligned}
$$

The above results now enable us to prove directly the finer structure of the Euclidean Teodorescu inversion, already observed above.

Proposition 2 The Teodorescu inversion formulae take the form

(i) $\partial_{\underline{X}} \bullet T=\mathbf{1}_{\Omega}$

(ii) $\partial_{\underline{X}} \wedge T=0$

Proof.

Take a function $u \in C_{1}(\bar{\Omega})$. Then

$$
\begin{aligned}
\partial_{\underline{X}} & \bullet T[u](\underline{X})=-\sum_{\alpha=1}^{m} \partial_{X_{\alpha}} T_{\alpha}[u](\underline{X}) \\
& =-\sum_{\alpha=1}^{m}\left(-\frac{1}{m} u(\underline{X})-\frac{1}{a_{m}} \mathrm{Fp} \int_{\Omega} \frac{u(\underline{Y})}{|\underline{X}-\underline{Y}|^{m}} d V(\underline{Y})+\frac{m}{a_{m}} \mathrm{Fp}_{\Omega} \frac{\left(\mathrm{X}_{\alpha}-\mathrm{Y}_{\alpha}\right)^{2}}{|\underline{\mathrm{X}}-\underline{\mathrm{Y}}|^{\mathrm{m}+2}} \mathrm{u}(\underline{\mathrm{Y}}) \mathrm{dV}(\underline{\mathrm{Y}})\right) \\
& =u(\underline{X})+\frac{m}{a_{m}} \mathrm{Fp} \int_{\Omega} \frac{u(\underline{Y})}{|\underline{X}-\underline{Y}|^{m}} d V(\underline{Y})-\frac{m}{a_{m}} \mathrm{Fp} \int_{\Omega} \sum_{\alpha=1}^{\mathrm{m}} \frac{\left(\underline{\mathrm{X}}-\mathrm{Y}_{\alpha}\right)^{2}}{|\underline{\mathrm{X}}|^{\mathrm{m}+2}} \mathrm{u}(\underline{\mathrm{Y}}) \mathrm{dV}(\underline{\mathrm{Y}})
\end{aligned}
$$

from which (i) follows since the last two terms cancel. Furthermore

$$
\partial_{\underline{X}} \wedge T[u](\underline{X})=\sum_{\alpha<\beta} e_{\alpha} e_{\beta}\left(\partial_{X_{\alpha}} T_{\beta}[u](\underline{X})-\partial_{X_{\beta}} T_{\alpha}[u](\underline{X})\right)
$$

which is zero since, in view of (8), each term vanishes. This yields (ii).

\section{The Hermitean Teodorescu operators}

As the kernel of the Teodorescu integral operator is the fundamental solution of the Dirac operator, subject to translation, we start by constructing the Hermitean counterparts of the pair of fundamental solutions $(E, E \mid)$ to the Dirac operators $\left(\partial_{\underline{X}}, \partial_{\underline{X} \mid}\right)$; they are given by

$$
\mathcal{E}=-(E+i E \mid), \quad \mathcal{E}^{\dagger}=(E-i E \mid)
$$


or, more explicitly:

$$
\begin{aligned}
& \mathcal{E}(\underline{z})=\frac{2}{a_{2 n}} \frac{\underline{z}}{|\underline{z}|^{2 n}}=\sum_{j=1}^{n} \mathfrak{f}_{j}\left(\frac{2}{a_{2 n}} \frac{z_{j}}{r^{2 n}}\right)=\sum_{j=1}^{n} \mathfrak{f}_{j} \mathcal{E}_{j} \\
& \mathcal{E}^{\dagger}(\underline{z})=\frac{2}{a_{2 n}} \frac{\underline{z}^{\dagger}}{|\underline{z}|^{2 n}}=\sum_{j=1}^{n} \mathfrak{f}_{j}^{\dagger}\left(\frac{2}{a_{2 n}} \frac{z_{j}^{c}}{r^{2 n}}\right)=\sum_{j=1}^{n} \mathfrak{f}_{j}^{\dagger} \mathcal{E}_{j}^{c}
\end{aligned}
$$

However, they are not the fundamental solutions to the respective Hermitean Dirac operators $\partial_{\underline{z}}$ and $\partial_{\underline{z}^{\dagger}}$. To show this we first rephrase the results of Lemma 2 to the Hermitean case.

Lemma 3 For all $j, k=1, \ldots, n, j \neq k$, one has in distributional sense

(i) $\partial_{z_{j}} \mathcal{E}_{j}=\frac{1}{n} \delta+\frac{2}{a_{2 n}} \mathrm{Fp} \frac{1}{r^{2 n}}-\frac{2 n}{a_{2 n}} \mathrm{Fp} \frac{\left|\mathrm{z}_{\mathrm{j}}\right|^{2}}{\mathrm{r}^{2 \mathrm{n}+2}}$

(ii) $\partial_{z_{k}} \mathcal{E}_{j}=-\frac{2 n}{a_{2 n}} \mathrm{Fp} \frac{z_{j} z_{k}^{c}}{r^{2 n+2}}$

(iii) $\partial_{z_{j}^{c}} \mathcal{E}_{j}^{c}=\frac{1}{n} \delta+\frac{2}{a_{2 n}} \mathrm{Fp} \frac{1}{r^{2 n}}-\frac{2 n}{a_{2 n}} \mathrm{Fp} \frac{\left|\mathrm{z}_{\mathrm{j}}\right|^{2}}{\mathrm{r}^{2 \mathrm{n}+2}}$

(iv) $\partial_{z_{k}^{c}} \mathcal{E}_{j}^{c}=-\frac{2 n}{a_{2 n}} \mathrm{Fp} \frac{z_{k} z_{j}^{c}}{r^{2 n+2}}$

(v) $\partial_{z_{k}} \mathcal{E}_{j}^{c}=\partial_{z_{j}} \mathcal{E}_{k}^{c}=-\frac{2 n}{a_{2 n}} \mathrm{Fp} \frac{z_{j}^{c} z_{k}^{c}}{r^{2 n+2}}$

(vi) $\partial_{z_{k}^{c}} \mathcal{E}_{j}=\partial_{z_{j}^{c}} \mathcal{E}_{k}=-\frac{2 n}{a_{2 n}} \mathrm{Fp} \frac{z_{j} z_{k}}{r^{2 n+2}}$

A rather straightforward computation then leads to the following results; they involve the so-called Spin-Euler operator $\beta$ which is para-bivector valued and given by

$$
\beta=\sum_{j=1}^{n} \mathfrak{f}_{j}^{\dagger} \mathfrak{f}_{j}=\frac{n}{2}+\frac{i}{2} \sum_{j=1}^{n} e_{j} e_{n+j}
$$

Proposition 3 One has in distributional sense

(i) $\partial_{\underline{z}} \mathcal{E}(\underline{z})=\frac{1}{n} \beta \delta\left(\underline{z}, \underline{z}^{\dagger}\right)+\frac{2}{a_{2 n}} \beta \mathrm{Fp} \frac{1}{r^{2 n}}-\frac{2}{a_{2 n}} n \mathrm{Fp} \frac{\underline{z}^{\dagger} \underline{z}}{r^{2 n+2}}$

(ii) $\partial_{\underline{z}^{\dagger}} \mathcal{E}(\underline{z})=0$

(iii) $\partial_{\underline{z}} \mathcal{E}^{\dagger}(\underline{z})=0$

(iv) $\partial_{\underline{z}^{\dagger}} \mathcal{E}^{\dagger}(\underline{z})=\frac{1}{n}(n-\beta) \delta\left(\underline{z}, \underline{z}^{\dagger}\right)+\frac{2}{a_{2 n}}(n-\beta) \operatorname{Fp} \frac{1}{r^{2 n}}-\frac{2}{a_{2 n}} n \operatorname{Fp} \frac{\underline{z} \underline{z}^{\dagger}}{r^{2 n+2}}$

(v) $\partial_{\underline{z}} \mathcal{E}(\underline{z})+\partial_{\underline{z}^{\dagger}} \mathcal{E}^{\dagger}(\underline{z})=\delta\left(\underline{z}, \underline{z}^{\dagger}\right)$

For a more thorough study of the fundamental solutions to the Euclidean and Hermitean Dirac operators and in particular their behaviour under the action of the operators $\partial_{\underline{z}} \bullet, \partial_{\underline{z}} \bullet$, $\partial_{\underline{z}} \wedge$ and $\partial_{\underline{z}^{\dagger}} \wedge$, we refer the reader to [17]. These operators originate by splitting the Clifford algebra product - which is always tacitly involved when a Clifford vector operator acts on a Clifford vector function - into its scalar or "dot" part and its bivector or "wedge" part. Moreover these operators are the counterparts in Hermitean Clifford analysis to the wellknown operators $\bar{\partial}, \bar{\partial}^{*}, \partial$ and $\partial^{*}$, the bar here denoting complex conjugation, for complex differential forms (see [19]). Here let us just mention that

$$
\partial_{\underline{z}} \bullet \mathcal{E}(\underline{z})=\frac{1}{2} \delta\left(\underline{z}, \underline{z}^{\dagger}\right)=\partial_{\underline{z}^{\dagger}} \bullet \mathcal{E}^{\dagger}(\underline{z})
$$

whence $2 \mathcal{E}(\underline{z})$ may be interpreted as a fundamental solution of the operator $\left(\partial_{\underline{z}} \cdot\right)$ and $2 \mathcal{E}^{\dagger}(\underline{z})$ as a fundamental solution of $\left(\partial_{\underline{z}^{\dagger}}\right)$.

Moreover on the basis of the results of Proposition 3, an important result was obtained in [10], by considering the particular circulant $(2 \times 2)$ matrices

$$
\mathcal{D}_{\left(\underline{z}, \underline{z}^{\dagger}\right)}=\left(\begin{array}{ll}
\partial_{\underline{z}} & \partial_{z^{\dagger}} \\
\partial_{\underline{z}^{\dagger}} & \partial_{\underline{z}}
\end{array}\right), \quad \mathcal{E}=\left(\begin{array}{cc}
\mathcal{E} & \mathcal{E}^{\dagger} \\
\mathcal{E}^{\dagger} & \mathcal{E}
\end{array}\right) \quad \text { and } \quad \delta=\left(\begin{array}{cc}
\delta & 0 \\
0 & \delta
\end{array}\right)
$$


Indeed, it then holds that

$$
\mathcal{D}_{\left(\underline{z}, \underline{z}^{\dagger}\right)} \mathcal{E}(\underline{z})=\delta(\underline{z})
$$

meaning that $\mathcal{E}$ may be considered as a fundamental solution of $\mathcal{D}_{\left(\underline{z}, \underline{z}^{\dagger}\right.}$. It is precisely this simple observation which has inspired the idea of a matrix approach to establish a Cauchy integral formula in the Hermitean setting. Also note that the Dirac matrix $\mathcal{D}_{\left(\underline{z}, \underline{z}^{\dagger}\right)}$ in some sense factorizes the Laplacian, since

$$
4 \mathcal{D}_{\left(\underline{z}, \underline{z}^{\dagger}\right)}\left(\mathcal{D}_{\left(\underline{z}, \underline{z}^{\dagger}\right)}\right)^{\dagger}=\left(\begin{array}{cc}
\Delta_{2 n} & 0 \\
0 & \Delta_{2 n}
\end{array}\right)=\Delta_{2 n}
$$

Now, in order to establish a Hermitean version of the Teodorescu inversion formula, we repeat the above procedure for the Euclidean Teodorescu operators, making suitable complex linear combinations:

$$
\begin{aligned}
& \mathcal{T}^{(1)}[(\cdot)](\underline{z})=(-1)^{\frac{n(n+1)}{2}}(2 i)^{n}(-T-i T \mid)=-\int_{\Omega} \mathcal{E}(\underline{w}-\underline{z})(\cdot)(\underline{w}) d W \\
& \mathcal{T}^{(2)}[(\cdot)](\underline{z})=(-1)^{\frac{n(n+1)}{2}}(2 i)^{n}(T-i T \mid)=-\int_{\Omega} \mathcal{E}^{\dagger}(\underline{w}-\underline{z})(\cdot)(\underline{w}) d W
\end{aligned}
$$

where we have used the notations $\underline{w}$ and $\underline{w}^{\dagger}$ for the Hermitean variables associated to the variables $\underline{Y}$ and $\underline{Y}$ and have put

$$
d W=\left(d w_{1} \wedge d w_{1}^{c}\right) \wedge\left(d w_{2} \wedge d w_{2}^{c}\right) \wedge \cdots \wedge\left(d w_{n} \wedge d w_{n}^{c}\right)=(-1)^{\frac{n(n+1)}{2}}(2 i)^{n} d V(\underline{Y})
$$

Using these definitions, we arrive at the desired result.

Theorem 1 (Hermitean Teodorescu inversion) Let $\Omega$ be a bounded domain in $\mathbb{R}^{2 n} \cong$ $\mathbb{C}^{n}$ with smooth boundary $\partial \Omega$, and let $u \in C_{1}(\bar{\Omega})$. Then $\mathcal{T}^{(1)}[u]$ and $\mathcal{T}^{(2)}[u]$ are in $C_{1}(\Omega)$ and they satisfy in $\Omega$

(i) $\partial_{\underline{z}} \mathcal{T}^{(1)}[u]+\partial_{\underline{z}^{\dagger}} \mathcal{T}^{(2)}[u]=(-1)^{\frac{n(n+1)}{2}}(2 i)^{n} u$

(ii) $\partial_{\underline{z}^{\dagger}} \mathcal{T}^{(1)}[u]+\partial_{\underline{z}} \mathcal{T}^{(2)}[u]=0$

Proof.

We only prove (i), the proof of (ii) being similar. Temporarily denoting $C=(-1)^{\frac{n(n+1)}{2}}(2 i)^{n}$ we have

$$
\begin{aligned}
\partial_{\underline{z}} \mathcal{T}^{(1)}[u]+\partial_{\underline{z}^{\dagger}} \mathcal{T}^{(2)}[u] & =-\frac{C}{4}\left(\partial_{\underline{X}}-i \partial_{\underline{X} \mid}\right)(-T[u]-i T \mid[u])+\frac{C}{4}\left(\partial_{\underline{X}}+i \partial_{\underline{X} \mid}\right)(T[u]-i T \mid[u]) \\
& =\frac{C}{4}\left(2 \partial_{\underline{X}} T[u]+2 \partial_{\underline{X}}|T|[u]\right)=\frac{C}{2}(u+u)=C u
\end{aligned}
$$

The results of the foregoing theorem may be written in matrix form as

$$
\left(\begin{array}{cc}
\partial_{\underline{z}} & \partial_{\underline{z}^{\dagger}} \\
\partial_{\underline{z}^{\dagger}} & \partial_{\underline{z}}
\end{array}\right)\left(\begin{array}{ll}
\mathcal{T}^{(1)}[u] & \mathcal{T}^{(2)}[u] \\
\mathcal{T}^{(2)}[u] & \mathcal{T}^{(1)}[u]
\end{array}\right)=(-1)^{\frac{n(n+1)}{2}}(2 i)^{n}\left(\begin{array}{cc}
u & 0 \\
0 & u
\end{array}\right)
$$

or still more explicitly

$$
-\mathcal{D}_{\left(\underline{z}, \underline{z}^{\dagger}\right)} \int_{\Omega} \mathcal{E}(\underline{w}-\underline{z})\left(\begin{array}{cc}
u(\underline{w}) & 0 \\
0 & u(\underline{w})
\end{array}\right) d W=(-1)^{\frac{n(n+1)}{2}}(2 i)^{n}\left(\begin{array}{cc}
u(\underline{w}) & 0 \\
0 & u(\underline{w})
\end{array}\right)
$$

which formally mimics the traditional Teodorescu inversion formula (4).

Our aim now is to refine these Hermitean Teodorescu inversion formulae, first with respect to the action of the associated Hermitean Dirac operators $\partial_{\underline{z}} \bullet, \partial_{z^{\dagger}} \bullet, \partial_{\underline{z}} \wedge$ and $\partial_{z^{\dagger}} \wedge$, next with respect to the individual action of the Hermitean Dirac operators on both Hermitean Teodorescu operators. To that end we first calculate the inversion formulae at the component level, putting for each function $u \in C_{1}(\Omega)$

$$
\begin{aligned}
& \mathcal{T}_{j}^{(1)}[u]=\int_{\Omega} \mathcal{E}_{j}(\underline{z}-\underline{w}) u(\underline{w}) d W \\
& \mathcal{T}_{j}^{(2)}[u]=\int_{\Omega} \mathcal{E}_{j}^{c}(\underline{z}-\underline{w}) u(\underline{w}) d W
\end{aligned}
$$


Proposition 4 For a function $u \in C_{1}(\bar{\Omega})$ and for $j, k=1, \ldots, n, j \neq k$, one has

(i) $\partial_{z_{j}} \int_{\Omega} \mathcal{E}_{j}(\underline{z}-\underline{w}) u(\underline{w}) d W=(-1)^{\frac{n(n+1)}{2}}(2 i)^{n} \frac{1}{n} u(\underline{z})+\mathrm{pv} \int_{\Omega} \partial_{z_{j}} \mathcal{E}_{j}(\underline{z}-\underline{w}) u(\underline{w}) d W$

(ii) $\partial_{z_{k}} \int_{\Omega} \mathcal{E}_{j}(\underline{z}-\underline{w}) u(\underline{w}) d W=\mathrm{pv} \int_{\Omega} \partial_{z_{k}} \mathcal{E}_{j}(\underline{z}-\underline{w}) u(\underline{w}) d W$

(iii) $\partial_{z_{j}^{c}} \int_{\Omega} \mathcal{E}_{j}^{c}(\underline{z}-\underline{w}) u(\underline{w}) d W=(-1)^{\frac{n(n+1)}{2}}(2 i)^{n} \frac{1}{n} u(\underline{z})+\mathrm{pv} \int_{\Omega} \partial_{z_{j}^{c}} \mathcal{E}_{j}^{c}(\underline{z}-\underline{w}) u(\underline{w}) d W$

(iv) $\partial_{z_{k}^{c}} \int_{\Omega} \mathcal{E}_{j}^{c}(\underline{z}-\underline{w}) u(\underline{w}) d W=\operatorname{pv} \int_{\Omega} \partial_{z_{k}^{c}} \mathcal{E}_{j}^{c}(\underline{z}-\underline{w}) u(\underline{w}) d W$

(v) $\partial_{z_{k}} \int_{\Omega} \mathcal{E}_{j}^{c}(\underline{z}-\underline{w}) u(\underline{w}) d W=\mathrm{pv} \int_{\Omega} \partial_{z_{k}} \mathcal{E}_{j}^{c}(\underline{z}-\underline{w}) u(\underline{w}) d W$

(vi) $\partial_{z_{k}^{c}} \int_{\Omega} \mathcal{E}_{j}(\underline{z}-\underline{w}) u(\underline{w}) d W=\mathrm{pv} \int_{\Omega} \partial_{z_{k}^{c}} \mathcal{E}_{j}(\underline{z}-\underline{w}) u(\underline{w}) d W$

Proof.

The proofs of all these relations being similar, we only prove the first one. To this end we extend the function $u \in C_{1}(\bar{\Omega})$ by zero in $\mathbb{R}^{2 n} \backslash \bar{\Omega}$ to $\widetilde{u}$ and we consider this extension $\widetilde{u}$ as a compactly supported distribution. Denoting temporarily $C=(-1)^{\frac{n(n+1)}{2}}(2 i)^{n}$ we have

$\partial_{z_{j}} \mathcal{T}_{j}^{(1)}[\widetilde{u}]=C \partial z_{j}\left(\mathcal{E}_{j} * \widetilde{u}\right)=C\left(\partial z_{j} \mathcal{E}_{j}\right) * \widetilde{u}=C\left(\frac{1}{n} \delta+\frac{2}{a_{2 n}} \mathrm{Fp} \frac{1}{r^{2 n}}-\frac{2 n}{a_{2 n}} \mathrm{Fp} \frac{\left|z_{j}\right|^{2}}{r^{2 n+2}}\right) * \widetilde{u}$

which, restricting to $\Omega$ also may be written as

$$
\partial_{z_{j}} \int_{\Omega} \mathcal{E}_{j}(\underline{z}-\underline{w}) u(\underline{w}) d W=\frac{C}{n} u(\underline{z})+\mathrm{pv} \int_{\Omega} \partial_{z_{j}} \mathcal{E}_{j}(\underline{z}-\underline{w}) u(\underline{w}) d W
$$

since for $\underline{w} \neq \underline{z}$

$$
\partial_{z_{j}} \mathcal{E}_{j}(\underline{z}-\underline{w})=\frac{2}{a_{2 n}} \frac{1}{\rho^{2 n}}-\frac{2 n}{a_{2 n}} \frac{\left|z_{j}-w_{j}\right|^{2}}{\rho^{2 n+2}}
$$

with $\rho=|\underline{z}-\underline{w}|$.

By making the appropriate combinations of the formulae obtained in the forgoing proposition we obtain the Hermitean Teodorescu Inversion Formulae for the associated Hermitean Dirac operators $\partial_{\underline{z}} \bullet, \partial_{\underline{z}^{\dagger}} \bullet, \partial_{\underline{z}} \wedge$ and $\partial_{\underline{z}^{\dagger}} \wedge$.

Theorem 2 For a function $u \in C_{1}(\bar{\Omega})$ one has

$$
\begin{aligned}
\partial_{\underline{z}} \bullet \mathcal{T}^{(1)}[u](\underline{z}) & =\frac{1}{2}(-1)^{\frac{n(n+1)}{2}}(2 i)^{n} u(\underline{z}) \\
\partial_{\underline{z}} \wedge \mathcal{T}^{(1)}[u](\underline{z}) & =\frac{1}{n}(-1)^{\frac{n(n+1)}{2}}(2 i)^{n}\left(\sum_{j=1}^{n} \mathfrak{f}_{j}^{\dagger} \wedge \mathfrak{f}_{j}\right) u(\underline{z})+\operatorname{pv} \int_{\Omega} \partial_{\underline{z}} \mathcal{E}(\underline{z}-\underline{w}) u(\underline{w}) d W \\
\partial_{\underline{z}^{\dagger}} \bullet \mathcal{T}^{(1)}[u](\underline{z}) & =0 \\
\partial_{\underline{z}^{\dagger}} \wedge \mathcal{T}^{(1)}[u](\underline{z}) & =0
\end{aligned}
$$

and

$$
\begin{aligned}
\partial_{\underline{z}} \bullet \mathcal{T}^{(2)}[u](\underline{z}) & =0 \\
\partial_{\underline{z}} \wedge \mathcal{T}^{(2)}[u](\underline{z}) & =0 \\
\partial_{\underline{z}^{\dagger}} \bullet \mathcal{T}^{(2)}[u](\underline{z}) & =\frac{1}{2}(-1)^{\frac{n(n+1)}{2}}(2 i)^{n} u(\underline{z}) \\
\partial_{\underline{z}^{\dagger}} \wedge \mathcal{T}^{(2)}[u](\underline{z}) & =\frac{1}{n}(-1)^{\frac{n(n+1)}{2}}(2 i)^{n}\left(\sum_{j=1}^{n} \mathfrak{f}_{j} \wedge \mathfrak{f}_{j}^{\dagger}\right) u(\underline{z})+\operatorname{pv} \int_{\Omega} \partial_{\underline{z}^{\dagger}} \mathcal{E}^{\dagger}(\underline{z}-\underline{w}) u(\underline{w}) d W
\end{aligned}
$$


Proof.

The proofs of all these relations being similar, we only prove the second one. Keeping the same notations as above we have

$$
\begin{aligned}
& \partial_{\underline{z}} \wedge \mathcal{T}^{(1)}[u](\underline{z})=\sum_{k=1}^{n} \sum_{j=1}^{n} \mathfrak{f}_{k}^{\dagger} \wedge \mathfrak{f}_{j} \partial_{z_{k}} \int_{\Omega} \mathcal{E}_{j}(\underline{z}-\underline{w}) u(\underline{w}) d W \\
& =\sum_{j=1}^{n} \mathfrak{f}_{j}^{\dagger} \wedge \mathfrak{f}_{j}\left(\frac{C}{n} u(\underline{z})+\mathrm{pv} \int_{\Omega} \partial_{z_{j}} \mathcal{E}_{j}(\underline{z}-\underline{w}) u(\underline{w}) d W\right)+\sum_{j \neq k} \mathfrak{f}_{k}^{\dagger} \wedge \mathfrak{f}_{j} \mathrm{pv} \int_{\Omega} \partial_{z_{k}} \mathcal{E}_{j}(\underline{z}-\underline{w}) u(\underline{w}) d W \\
& =\frac{C}{n}\left(\sum_{j=1}^{n} \mathfrak{f}_{j}^{\dagger} \wedge \mathfrak{f}_{j}\right) u(\underline{z})+\mathrm{pv} \int_{\Omega} \sum_{j=1}^{n} \sum_{k=1}^{n} \mathfrak{f}_{k}^{\dagger} \wedge \mathfrak{f}_{j} \partial_{z_{k}} \mathcal{E}_{j}(\underline{z}-\underline{w}) u(\underline{w}) d W \\
& =\frac{C}{n}\left(\sum_{j=1}^{n} \mathfrak{f}_{j}^{\dagger} \wedge \mathfrak{f}_{j}\right) u(\underline{z})+\mathrm{pv} \int_{\Omega} \partial_{\underline{z}} \wedge \mathcal{E}(\underline{z}-\underline{w}) u(\underline{w}) d W
\end{aligned}
$$

from which the desired result follows since, by (11), $\partial_{\underline{z}} \mathcal{E}=\partial_{\underline{z}} \wedge \mathcal{E}$ as long as $\underline{z} \neq \underline{w}$.

Corollary 2 One has

$$
\begin{aligned}
& \text { (i) } \partial_{\underline{z}} \bullet \mathcal{T}^{(1)}[u](\underline{z})+\partial_{\underline{z}^{\dagger}} \bullet \mathcal{T}^{(2)}[u](\underline{z})=\frac{1}{2}(-1)^{\frac{n(n+1)}{2}}(2 i)^{n} u(\underline{z}) \\
& \text { (ii) } \partial_{\underline{z}} \wedge \mathcal{T}^{(1)}[u](\underline{z})+\partial_{\underline{z}^{\dagger}} \wedge \mathcal{T}^{(2)}[u](\underline{z})=0
\end{aligned}
$$

Finally, making the appropriate combinations of the formulae of the foregoing theorem, we obtain expressions for the action of the Hermitean Dirac operators on the Hermitean Teodorescu operators.

Proposition 5 For a function $u \in C_{1}(\bar{\Omega})$ one has

$$
\begin{aligned}
& \text { (i) } \partial_{\underline{z}} \mathcal{T}^{(1)}[u]=(-1)^{\frac{n(n+1)}{2}}(2 i)^{n} \frac{1}{n} \beta u+P . V \cdot \int_{\Omega} \partial_{\underline{z}} \mathcal{E}(\underline{z}-\underline{w}) u(\underline{w}) d W \\
& \text { (ii) } \partial_{\underline{z}^{\dagger}} \mathcal{T}^{(1)}[u]=0 \\
& \text { (iii) } \partial_{\underline{z}} \mathcal{T}^{(2)}[u]=0 \\
& \text { (iv) } \partial_{\underline{z}^{\dagger}} \mathcal{T}^{(2)}[u]=(-1)^{\frac{n(n+1)}{2}}(2 i)^{n}\left(1-\frac{1}{n} \beta\right) u+P . V \cdot \int_{\Omega} \partial_{\underline{z}^{\dagger}} \mathcal{E}^{\dagger}(\underline{z}-\underline{w}) u(\underline{w}) d W
\end{aligned}
$$

Remark 3 Note that formula (i) of Theorem 1 follows from formulae (i) and (iv) of Proposition 5 due to formula (v) of Proposition 3. Note also that formulae (ii) and (iii) of Proposition 5 are stronger than formula (ii) of Theorem 1.

\section{The several complex variables case}

In this section we will restrict ourselves to complex valued functions $f: \mathbb{R}^{m} \cong \mathbb{C}^{2 n} \rightarrow \mathbb{C}$. Such functions which a priori are functions of the real variables $\left(x_{1}, \ldots, x_{n}, y_{1}, \ldots, y_{n}\right)$ become functions of $n$ complex variables and their complex conjugates: $f\left(z_{1}, \ldots, z_{n}, z_{1}^{c}, \ldots, z_{n}^{c}\right)$ or in shorthand $f\left(\underline{z}, \underline{z}^{\dagger}\right)$. It is only when a function is holomorphic that it becomes function of the variables $\left(z_{1}, \ldots, z_{n}\right)$ only. Naturally all the results obtained in the foregoing section apply to such scalar valued functions.

When concentrating on Proposition 4, we see that, quite naturally, only formulae (iii) and (iv) are to be found in the literature on several complex variables, see e.g. [25, (1.11)], since there the focus is on holomorphic functions and the related Cauchy-Riemann operators $\partial_{z_{j}^{c}}, j=1, \ldots, n$, while there is not one unique differential operator defining multidimensional holomorphy. The mirror formulae (i) and (ii) involving the conjugate Cauchy-Riemann operators $\partial_{z_{j}^{c}}, j=1, \ldots, n$ are then tacitly assumed. However the formulae from Proposition 4 do not really deserve the qualification of Teodorescu inversion. It becomes interesting when summing them up, leading to the results already contained in Theorem 2 :

$$
\partial_{\underline{z}} \bullet \mathcal{T}^{(1)}[f]=\frac{1}{2} \sum_{j=1}^{n} \partial_{z_{j}} \int_{\Omega} \mathcal{E}_{j}(\underline{z}-\underline{w}) f(\underline{w}) d W=\frac{1}{2}(-1)^{\frac{n(n+1)}{2}}(2 i)^{n} f(\underline{z})
$$




$$
\sum_{j=1}^{n} \partial_{z_{j}} \int_{\Omega} \frac{2}{a_{2 n}} \frac{z_{j}-w_{j}}{\rho^{2 n}} f(\underline{w}) d W=(-1)^{\frac{n(n+1)}{2}}(2 i)^{n} f(\underline{z})
$$

and

$$
\partial_{\underline{z}^{\dagger}} \bullet \mathcal{T}^{(2)}[f]=\frac{1}{2} \sum_{j=1}^{n} \partial_{z_{j}^{c}} \int_{\Omega} \mathcal{E}_{j}^{c}(\underline{z}-\underline{w}) f(\underline{w}) d W=\frac{1}{2}(-1)^{\frac{n(n+1)}{2}}(2 i)^{n} f(\underline{z})
$$

or

$$
\sum_{j=1}^{n} \partial_{z_{j}^{c}} \int_{\Omega} \frac{2}{a_{2 n}} \frac{z_{j}^{c}-w_{j}^{c}}{\rho^{2 n}} f(\underline{w}) d W=(-1)^{\frac{n(n+1)}{2}}(2 i)^{n} f(\underline{z})
$$

expressing the fact that $\mathcal{T}^{(1)}$ and $\mathcal{T}^{(2)}$ indeed are the right inverses of the associated Dirac operators $\partial_{\underline{z}} \bullet$ and $\partial_{\underline{z} \dagger} \bullet$ respectively.

As was expected the involved integral kernels are not (anti-)holomorphic, but still harmonic, since they are the components of the fundamental solutions of both Hermitean Dirac operators. And moreover they do coincide with the ones appearing in the Martinelli-Bochner representation formula for holomorphic functions (2) and its anti-holomorphic counterpart.

\section{References}

[1] R. Abreu Blaya, J. Bory Reyes, F. Brackx, B. De Knock, H. De Schepper, D. Pena Pena, F. Sommen, Hermitean Cauchy integral decomposition of continuous functions on hypersurfaces, Bound. Value Probl. 2009, 2009, ID 425256.

[2] R. Abreu Blaya, J. Bory Reyes, D. Pena Pena, F. Sommen, A boundary value problem for Hermitian monogenic functions, Bound. Value Probl. 2008, 2008, ID 385874.

[3] F. Brackx , J. Bureš, H. De Schepper, D. Eelbode, F. Sommen and V. Souček, Fundaments of Hermitean Clifford Analysis. Part I: Complex structure, Compl. Anal. Oper. Theory 1(3), 2007, 341-365.

[4] F. Brackx, J. Bureš, H. De Schepper, D. Eelbode, F. Sommen and V. Souček, Fundaments of Hermitean Clifford Analysis. Part II: Splitting of h-monogenic equations, Complex Var. Elliptic Eq. 52(10-11), 2007, 1063-1079.

[5] F. Brackx, B. De Knock and H. De Schepper, A specific family of Clifford distributions. In: L. Son, W. Tutschke, S. Jain (eds.), Methods of complex and Clifford analysis, SAS Int. Publ., Delhi, 2004, 215-227.

[6] F. Brackx, B. De Knock and H. De Schepper, Multi-vector spherical monogenics, spherical means and distributions in Clifford analysis, Acta Math. Sin. 21(5), 2005, 1197-1208.

[7] F. Brackx, B. De Knock and H. De Schepper, On the Fourier spectra of distributions in Clifford analysis, Chin. Ann. Math. 27B(5), 2006, 495-506.

[8] F. Brackx, B. De Knock, H. De Schepper and D. Eelbode, A Calculus Scheme for Clifford Distributions, Tokyo J. Math. 29(2), 2006, 495-513.

[9] F. Brackx, B. De Knock, H. De Schepper and F. Sommen, Distributions in Clifford Analysis: an Overview. In: S.-L. Eriksson (ed.), Clifford Analysis and Applications Proceedings of the Summer School, Tampere 2004, Tampere University of Technology, Institute of Mathematics, Research Report No. 82, 2006, 59-73.

[10] F. Brackx, B. De Knock, H. De Schepper and F. Sommen, On Cauchy and MartinelliBochner integral formulae in Hermitean Clifford analysis, Bull. Braz. Math. Soc. 40(3), 2009, 395-416.

[11] F. Brackx, R. Delanghe and F. Sommen, Clifford Analysis, Pitman Publishers, BostonLondon-Melbourne, 1982.

[12] F. Brackx, R. Delanghe and F. Sommen, Spherical means, distributions and convolution operators in Clifford analysis, Chin. Ann. Math. 24B(2), 2003, 133-146.

[13] F. Brackx, R. Delanghe and F. Sommen, Spherical means and distributions in Clifford analysis. In: T. Qian, T. Hempfling, A. McIntosh and F. Sommen (eds.), Advances in analysis and geometry: new developments using Clifford algebras, Trends in Mathematics, Birkhäuser Verlag, Basel, 2004, 65-96. 
[14] F. Brackx, R. Delanghe and F. Sommen, Differential forms and/or multivector functions, Cubo 7(2), 2005, 139-169.

[15] F. Brackx, H. De Schepper, D. Eelbode and V. Souček, The Howe dual pair in Hermitean Clifford analysis, to appear in Rev. Mat. Iberoamericana.

[16] F. Brackx, H. De Schepper, M.E. Luna Elizarrars, M. Shapiro, Integral representation formulae in Hermitean Clifford analysis. In: K. Gürlebeck and C. Könke (eds.), Proceedings of the 18th International Conference on the Application of Computer Science and Mathematics in Architecture and Civil Engineering, Weimar, Germany, 2009 (cd-rom).

[17] F. Brackx, H. De Schepper, M.E. Luna-Elizarrarás and M. Shapiro, On fundamental solutions in Clifford analysis, to appear in Compl. Anal. Oper. Theory.

[18] F. Brackx, H. De Schepper and F. Sommen, The Hermitian Clifford analysis toolbox, Appl. Clifford Algebras 18(3-4), 2008, 451-487.

[19] F. Brackx, H. De Schepper and V. Souček, Differential Forms versus Multivector Functions in Hermitean Clifford Analysis, to appear in Cubo.

[20] R. Delanghe, F. Sommen and V. Souček, Clifford Algebra and Spinor-Valued Functions, Kluwer Academic Publishers, Dordrecht, 1992.

[21] J. Gilbert and M. Murray, Clifford Algebras and Dirac Operators in Harmonic Analysis, Cambridge University Press, Cambridge, 1991.

[22] K. Guerlebeck and W. Sproessig, Quaternionic and Clifford Calculus for Physicists and Engineers, Wiley, Chichester, 1998.

[23] K. Guerlebeck, K. Habetha and W. Sproessig, Holomorphic functions in the plane and ndimensional space (translated from the 2006 German original), Birkhäuser Verlag, Basel, 2008.

[24] S. Krantz, Function theory of several complex variables, 2nd edition, Wadsworth \& Brooks/Cole, Pacific Grove, 1992.

[25] A. Kytmanov, The Bochner-Martinelli integral and its applications, Birkhäuser Verlag, Basel, 1995.

[26] R. Rocha-Chavez, M. Shapiro and F. Sommen, Integral theorems for functions and differential forms in $\mathbb{C}_{m}$, Research Notes in Math. 428, Chapman \& Hall/CRC, New York, 2002.

[27] J. Ryan, Complexified Clifford analysis, Complex Variables: Theory \& Application 1, 1982, 119-149.

[28] I. Sabadini and F. Sommen, Hermitian Clifford analysis and resolutions, Math. Meth. Appl. Sci. 25 (16-18), 2002, 1395-1414. 